\title{
ON A VARIATION OF SANDS' METHOD
}

\author{
EVELYN E. OBAID \\ Department of Mathematics and Computer Science \\ San Jose State University \\ San Jose, California 95192 U.S.A. \\ (Received January 15, 1985 and in revised form March 20, 1986)
}

ABSTRACT. A subset of a of a finite additive abelian group $G$ is a Z-set if for all $a \varepsilon G$, naeG for all $n \varepsilon Z$. The group $G$ is called "Z-good" if in every factorization $G=$ $A \oplus B$, where $A$ and $B$ are $Z$-sets at least one factor is periodic. Otherwise $G$ is called "Z-bad."

The purpose of this paper is to investigate factorizations of finite ablian groups which arise from a variation of Sands' method. A necessary condition is given for a factorization $G=A \oplus B$, where $A$ and $B$ are Z-sets, to be obtained by this variation. An example is provided to show that this condition is not sufficient. It is also shown that in general all factorizations $G=A \odot B$, where $A$ and $B$ are Z-sets, of $a$ "Z-good" group do not arise from this variation of Sands' method.

KEY WORDS AND PHRASES. Finite abelian group, factorization, Z-set, good group, bad group.

1980 MATHEMATICS SUBJECT CLASSIFICATION CODES. 20K01, $20 \mathrm{~K} 25$.

I. INTRODUCTION.

Let $\mathrm{G}$ be a finite additive abelian group and let $\mathrm{A}$ and $\mathrm{B}$ be subsets of $\mathrm{G}$. If every element $g \in G$ can be uniquely represented in the form $g=a+b$, where $a \in A$, $\mathrm{b} \varepsilon \mathrm{B}$, then we write $\mathrm{G}=\mathrm{A} \oplus \mathrm{B}$ and call this a factorization of $\mathrm{G}$. A subset $A$ of $G$ is said to be periodic if there exists an element $g \neq 0$ such that $g+A=A$. Such an element $g$ is called a period of $A$. The set of all periods of $A$ together with $O$ forms a subgroup of $G$. A subset of $G$ is a Z-set if for all a $\varepsilon A$, na $\varepsilon A$ for all $n \varepsilon z$. We say $G$ is "good" ("Z-good") if in every factorization $G=A \oplus B$, where $A$ and $B$ are sets (Z-sets) at least one factor is periodic. Otherwise G is called "bad" ("Z-bad").

The problem of classifying a finite abelian group as elther "good" or "bad" arose from the solution of $\mathrm{G}$. Hajos [1] to a group-theoretical interpretation of a conjecture of H. Minkowski on homogeneous linear forms. Hajos [1-3], Redel [4-5], de Brui.jn [6-7], and Sands [8-11] have completely solved this problem of classification. C. Okuda 
[12] classified all finite abelian groups as either "Z-gooc" of "Z-bad," obtaining quite different results from the "good" - "bad" classification.

Sands [8] gave a method which yields all factorizations of a finite abelian "good" group. His method corrects one given previously by Hajos [2].

The purpose of this paper is to investigate factorizations of finite abelian groups which arise from a variation of Sands' method. A necessary condition is given for a factorization $\mathrm{G}=\mathrm{A}+\mathrm{B}$, where $\mathrm{A}$ and $\mathrm{B}$ are $\mathrm{Z}$-sets, to be obtained by this variation. An example is provided to show that this condition is not sufficient. It is also shown that in general all factorizations $G=A \%, B$, where $A$ and $B$ are Z-sets, of $a$ "Z-good" group do not arise from this variation of Sands' method.

2. PRELIMINARIES.

This section provides some basic unpublished results on Okuda's [12] "Z-good" "Z-bad" classification of finite abelian groups as well as an elementary result concerning factorizations $G=S F, A$, where $S$ is a subgroup of $G$ and $A$ is a Z-set. For completeness, we state Sands' Theorem on the factorizations of finite abelian "good" groups.

LEMMA 1 (Okuda [12]). A finite abelian group G is "Z-good" if and only if at least one Sylow p-subgroup of $G$ is "Z-good."

LEMMA 2 (Okuda [12]). Every cyclic group is "Z-gcod."

LEMMA 3 (Okuda [12]). If $G=A \odot B$, where $A$ and $B$ are Z-sets, then $A$ and $B$ are pure in $G$.

LEMMA 4 (Okuda [12]). Let $G$ be a group isomorphic to $z_{p}+z_{p}+z_{p}+z_{p}$, where $p$ is an odd prime. Let $\left\{a_{1}, a_{2}, b_{1}, b_{2}\right\}$ be a basis of $G$ and def 1 ne

$$
\begin{aligned}
& A=\left(<a_{1}, a_{2}>\left(<a_{2}>\right)\left(i<a_{2}+b_{2}\right\rangle,\right. \\
& \left.\left.\left.B=\left(<b_{1}, b_{2}\right\rangle \backslash \bigcup_{i=1}^{p-1}<b_{1}+i b_{2}>\right)\right) \backslash\left(i_{i=1}^{p-1}<b_{1}+i b_{2}+2 a_{2}\right\rangle\right) .
\end{aligned}
$$

Then $A$ and $B$ are non-periodic Z-sets and $G=A+B$.

PROPOSITION 1. Let $S$ be a subgroup of $G$. $G$ has a factorization $G=S+A, A$ a Z-set, if and only if $S$ is pure in $G$.

PROOF. This is a direct consequence of Lemma 3 and the fact that a pure subgroup of a finite abelian group $G$ is a direct summand of $G$.

THEOREM 1 (Sands [8]). Let $G$ be a finite abelian "rood" group. $G=A+B$ if and only if there exists subsets $H_{1}, H_{2}, \ldots, H_{n}$ such that $H_{i}+H_{i+1}+\ldots+H_{n}=$ $K_{i}$ is a subgroup of $G, 1 \leq i \leq n, K_{1}=G$, and

$$
\begin{aligned}
& A=\langle 0\rangle+\mathrm{H}_{1} \circ \mathrm{H}_{2}+\mathrm{H}_{3} \circ \mathrm{H}_{4}+\ldots, \\
& B=\langle 0\rangle \circ \mathrm{H}_{1}+\mathrm{H}_{2} \circ \mathrm{H}_{3}+\mathrm{H}_{4} \circ \ldots,
\end{aligned}
$$

where the notation $C \circ D$ indicates any of the sets formed by adding to each element of C some element of $D$. 
Let us note that the subgroups, $\mathrm{K}_{i}$, in Sands' Theorem yield the following series for $G$

$$
\mathrm{G}=\mathrm{K}_{1} \supset \mathrm{K}_{2} \supset \mathrm{K}_{3} \supset \ldots \supset \mathrm{K}_{\mathrm{n}} \supset \mathrm{K}_{\mathrm{n}+1}=\langle 0\rangle
$$

where $K_{i}=H_{i} \cong K_{i+1}, 1 \leq i \leq n, K_{n}=H_{n}$. We shall say that the factorization $G=$ $A \mp B$ arises from the above series if

$$
\begin{aligned}
& A=\mathrm{H}_{1}+\mathrm{H}_{3}+\ldots+\mathrm{h}_{2}+\mathrm{h}_{4}+\cdots, \\
& \mathrm{B}=\mathrm{H}_{2}+\mathrm{H}_{4} \pm \ldots+\mathrm{h}_{1}+\mathrm{h}_{3}+\cdots,
\end{aligned}
$$

where $H_{i}$ is a set of coset representatives for $K_{i}$ modulo $K_{i+1}$, and $h_{i} \varepsilon H_{i}, 1 \leq i \leq n$.

Factorizations which arise from the above series can be obtained from Sands' method if one computes $C \circ D$ by adding a fixed element of $D$ to the set $C$. However, as shown in Example 1, there are factorizations which are obtained from Sands' method which do not arise from the corresponding series of subgroups $K_{i}, 1 \leq i \leq n$.

EXAMPLE 1. Let $G$ be the cyclic group of order 81 . Consider the series $G=$ $K_{1}-K_{2}-K_{3} \supset K_{4}-j\langle 0\rangle$, where $K_{4}=\langle 27\rangle, K_{3}=\langle 9\rangle, K_{2}=\langle 3\rangle$. If we choose $\mathrm{H}_{3}=\{0,9,18\}, \mathrm{H}_{2}=\{0,3,6\}, \mathrm{H}_{1}=\{0,1,2\}$ then the following factorization, $\mathrm{G}=\mathrm{A} \mp \mathrm{F}$, can be obtained from Sands' method.

$$
\begin{aligned}
A & =\langle 0\rangle \pm \mathrm{H}_{1} \circ \mathrm{H}_{2} \mp \mathrm{H}_{3} \circ \mathrm{H}_{4}=\left(\mathrm{H}_{1} \mp \mathrm{H}_{3}\right) \circ \mathrm{H}_{4} \\
& =(\{0,1,2\} \nsubseteq\{0,9,18\}) \circ\{0,27,54\}=\{0,1,2,9,10,11,18,19,47\} \\
B & =\langle 0\rangle \circ \mathrm{H}_{1} \oplus \mathrm{H}_{2} \circ \mathrm{H}_{3} \mp \mathrm{H}_{4}=\mathrm{H}_{2} \pm \mathrm{H}_{4}=\{0,3,6\} \oplus\{0,27,54\} .
\end{aligned}
$$

Clearly for every choice of the sets $H_{i}, 1 \leq i \leq 3$, the set $A$ cannot be written in the form $\mathrm{A}=\mathrm{H}_{1} \mp \mathrm{H}_{3}+\mathrm{h}_{2}+\mathrm{h}_{4}$.

3. TRANSLATIONS.

THEOREM 2. Let $\mathrm{G}=\mathrm{A}+\mathrm{B}$ a factorization of $\mathrm{G}$ arising from the series $\mathrm{G}=$ $\mathrm{K}_{1}-\mathrm{K}_{2}-\cdots \mathrm{K}_{\mathrm{n}}-\langle 0\rangle$ with coset representatives $\mathrm{H}_{\mathrm{i}}$ so that $\mathrm{K}_{\mathrm{i}}=\mathrm{H}_{\mathrm{i}}+\mathrm{K}_{\mathrm{i}+1}$, $1 \leq i \leq n-1, K_{n}=H_{n}$. Then $G=A^{\prime}$ I $B^{\prime}$ with $A^{\prime}=A+g_{1}, B^{\prime}=B+g_{2}$,

$g_{1}, g_{2} \varepsilon G$ arises from the same series with coset representatives $H_{i}^{\prime}=H_{i}+u_{1}$, $u_{1} \in H_{i}, 1 \leq i \leq n$.

PROOF. We will proceed by induction on the length of the series, $n$. For $n=$ ?, we may assume $A=H_{1}+h_{2}, B=H_{2}+h_{1}, h_{i} \varepsilon H_{i}$, $i=1,2$. Suppose $G=A^{\prime}+B^{\prime}$ with $A^{\prime}=A+g_{1}, B^{\prime}=B+g_{2}, g_{1}, g_{2} \varepsilon G$. We can write $g_{1}=u_{1}+u_{2}, u_{1} \in H_{i}, i=1,2$. Since $G=\left(H_{1}+u_{1}\right) \mp H_{2}$ we have $h_{1}+g_{2}=z_{1}+u_{1}+z_{2}, z_{i} \in H_{i}, i=1$, Let $\mathrm{H}_{1}^{\prime}=\mathrm{H}_{1}+\mathrm{u}_{1}, \mathrm{H}_{2}^{\prime}=\mathrm{H}_{2}+\mathrm{u}_{2}$. Then

$$
\begin{aligned}
& A^{\prime}=A+g_{1}=H_{1}+u_{1}+h_{2}+u_{2}=H_{1}^{\prime}+h_{2}^{\prime}, \\
& B^{\prime}=B+g_{2}=H_{2}+z_{2}+z_{1}+u_{1}=H_{2}+u_{2}+z_{1}+u_{1}=H_{2}^{\prime}+h_{1}^{\prime},
\end{aligned}
$$

where we have used the fact that $\mathrm{H}_{2}=\mathrm{H}_{2}+\mathrm{u}_{2}=\mathrm{H}_{2}+\mathrm{z}_{2}$ since $\mathrm{H}_{2}+\mathrm{K}_{2}$ is a subgroup.

Let us assume the theorem is true for serles of length less than $n$. Let $G=$ $\mathrm{A}+\mathrm{B}$ be a factorization arising from a series of length $\mathrm{n}$, say $\mathrm{G}=\mathrm{B}+\mathrm{E}_{2}$, $\mathrm{K}_{1} \supset \mathrm{K}_{2} \ldots \ldots \mathrm{K}_{\mathrm{n}} \supset<0>$. We may assume that 


$$
\begin{aligned}
& A=H_{1} \oplus \mathrm{H}_{3} \oplus \ldots+\mathrm{h}_{2}+\mathrm{h}_{4}+\ldots, \\
& B=\mathrm{H}_{2} \oplus \mathrm{H}_{4} \oplus \ldots+\mathrm{h}_{1}+\mathrm{h}_{3}+\ldots .
\end{aligned}
$$

Define

$$
\begin{aligned}
& A_{1}=H_{3} \subsetneq H_{5} \oplus \ldots+h_{2}+h_{4}+\ldots, \\
& B_{1}=H_{2} \subsetneq H_{4} \oplus \ldots+h_{3}+h_{5}+\ldots,
\end{aligned}
$$

so that $A=A_{1} \Subset H_{1}, B=B_{1}+h_{1}$. Suppose $G=A^{\prime} € B^{\prime}$, where

$$
\begin{aligned}
& A^{\prime}=A+g_{1}, g_{1}=s_{1}+s_{2}, s_{1} \varepsilon H_{1}, s_{2} \varepsilon K_{2}, \\
& B^{\prime}=B+g_{2}, g_{2}=t_{1}+t_{2}, t_{1} \varepsilon H_{1}, t_{2} \varepsilon K_{2} .
\end{aligned}
$$

Setting $\mathrm{H}_{1}^{\prime}=\mathrm{H}_{1}+\mathrm{s}_{1}$ we have

$$
\begin{aligned}
& A^{\prime}=H_{1}^{\prime} \odot\left(A_{1}+s_{2}\right), \\
& B^{\prime}=\left(h_{1}+t_{1}\right)+\left(B_{1}+t_{2}\right)=h_{1}^{\prime}+\left(B_{1}+t_{2}+k_{2}\right),
\end{aligned}
$$

where $h_{1}+t_{1}=h_{1}+t_{1}-s_{1}+s_{1}=\tilde{h}_{1}+k_{2}+s_{1}=h_{1}^{\prime}+k_{2}, k_{2} \varepsilon k_{2}, \tilde{h}_{1} \varepsilon H_{1}$, and $h_{1}^{\prime}=\tilde{h}_{1}+s_{1} \varepsilon H_{1}^{\prime}$.

Note that $\mathrm{K}_{2}=\mathrm{A}_{1} \odot \mathrm{B}_{1}$ arises from the series $\mathrm{K}_{2} \supset \mathrm{K}_{3}-\ldots \supset \mathrm{K}_{\mathrm{n}}-<0>$. Therefore the factorization $K_{2}=\left(A_{1}+s_{2}\right) \oplus\left(B_{1}+t_{2}+k_{2}\right)$ arises from $\mathrm{K}_{2} \cdots \mathrm{K}_{3} \ldots \ldots \mathrm{K}_{\mathrm{n}}{ }^{-}<0>$ with coset representatives $H_{i}^{\prime}=H_{i}+u_{i}, u_{i} \varepsilon H_{i}, 2 \leq i<-n$, i.e.,

$$
\begin{aligned}
& A_{1}+s_{2}=H_{3}^{\prime}+H_{5}^{\prime}+\ldots+h_{2}^{\prime}+h_{4}^{\prime}+\ldots, \\
& B_{1}+t_{2}+k_{2}=H_{2}^{\prime}+H_{4}^{\prime}+\ldots+h_{3}^{\prime}+h_{5}^{\prime}+\ldots .
\end{aligned}
$$

Consequently we have

$$
\begin{aligned}
& A^{\prime}=H_{1}^{\prime}+\left(A_{1}+S_{2}\right)=H_{1}^{\prime}+H_{3}^{\prime} \oplus \ldots+h_{2}^{\prime}+h_{4}^{\prime}+\ldots, \\
& B^{\prime}=h_{1}^{\prime}+\left(B_{1}+t_{2}+k_{2}\right)=H_{2}^{\prime} \oplus H_{4}^{\prime}+\ldots+h_{1}^{\prime}+h_{3}^{\prime}+\ldots,
\end{aligned}
$$

which completes the proof.

THEOREM 3. Let $G=A \biguplus B$ be a factorization of $G$ arising from the series $G=$ $\mathrm{K}_{1} \supset \mathrm{K}_{2}-\ldots \supset \mathrm{K}_{\mathrm{n}}<0>$ with coset representatives $\mathrm{H}_{i}$ so that $\mathrm{K}_{i}=\mathrm{H}_{i} \oplus \mathrm{K}_{i+1}$, $1 \leq i \leq n-1, K_{n}=H_{n}$. Then each $H_{i}$ may be translated to obtain $H_{i}^{\prime}, 1 \leq i \leq n$, in such a way that $0 \varepsilon H_{i}, 1 \leq i \leq n$, and the factorization $G=A$ it $B$ arises from the original series with coset representatives $H_{i}^{\prime}, 1 \leq i \leq n, K_{n}=H_{n}^{\prime}$.

PROOF. We will use induction on the length of the series, $n$. For $n=2$, we may assume $A=H_{1}+h_{2}, B=H_{2}+h_{1}, h_{i} \varepsilon H_{i}$, $i=1,2$. We can write $0=y_{1}+y_{2}, y_{i} \varepsilon H_{i}$, $i=1,2$. Define $H_{1}^{\prime}=H_{1}+y_{2}, H_{2}^{\prime}=H_{2}$, and let $h_{1}^{\prime}=h_{1}+y_{2}$. Note that $H_{2}=H_{2}+y_{2}$ and $h_{2}-y_{2} \in H_{2}$ since $H_{2}=K_{2}$ is a subgroup. Thus,

$$
\begin{aligned}
& A=H_{1}+y_{2}+h_{2}-y_{2}=H_{1}^{\prime}+h_{2}^{\prime}, \\
& B=H_{2}+h_{1}=H_{2}+h_{1}+y_{2}=H_{2}^{\prime}+h_{1}^{\prime} .
\end{aligned}
$$


Let us assume the theorem is true for series of length less than $n$. Let the factorization $G=A \mp B$ arise from a series of length $n$, say, $\mathrm{G}=\mathrm{K}_{1} \mathrm{~K}_{2} \cdots \ldots \supset \mathrm{K}_{\mathrm{n}} \supset<0>$. We may assume that

$$
\begin{aligned}
& A=H_{1} \oplus \mathrm{H}_{3} \oplus \ldots+\mathrm{h}_{2}+\mathrm{h}_{4}+\ldots, \\
& B=\mathrm{H}_{2} \oplus \mathrm{H}_{4} \oplus \ldots+\mathrm{h}_{1}+\mathrm{h}_{3}+\ldots .
\end{aligned}
$$

Define

$$
\begin{aligned}
& A_{1}=H_{3} \oplus H_{5} \oplus \ldots+h_{2}+h_{4}+\ldots, \\
& B_{1}=H_{2} \oplus H_{4} \oplus \ldots+h_{3}+h_{5}+\ldots .
\end{aligned}
$$

so that $A=A_{1} \oplus H_{1}, B=B_{1}+h_{1}$, and $K_{2}+A_{1} \oplus B_{1}$.

We can write $0=y_{1}+y_{2}+\ldots+y_{n}, y_{i} \varepsilon H_{i}, 1 \leq i \leq n$. Set $x_{2}=y_{2}+y_{3}+\ldots$ $+\mathrm{y}_{\mathrm{n}}$. Then $\mathrm{x}_{2} \varepsilon \mathrm{K}_{2}$. Define $\mathrm{H}_{1}^{\prime}=\mathrm{H}_{1}+\mathrm{x}_{2}$ and let $\mathrm{h}_{1}=\mathrm{h}_{1}^{\prime}+\mathrm{z}_{2}, \mathrm{z}_{2} \varepsilon \mathrm{K}_{2}$. Note that $0 \in H_{1}^{\prime}$. We have $K_{2}=\left(A_{1}-x_{2}\right) \oplus\left(B_{1}+z_{2}\right)$. By Theorem 2 there exists coset representatives $\mathrm{H}_{2}^{\prime}, \mathrm{H}_{3}^{\prime}, \ldots, \mathrm{H}_{\mathrm{n}}^{\prime}$ translates of $\mathrm{H}_{2}, \mathrm{H}_{3}, \ldots, \mathrm{H}_{n}$ respectively such that

$$
\begin{aligned}
& A_{1}-x_{2}=H_{3}^{\prime} \oplus H_{5}^{\prime} \oplus \ldots+h_{2}^{\prime}+h_{4}^{\prime}+\ldots, \\
& B_{1}+z_{2}=H_{2}^{\prime} \oplus H_{4}^{\prime} \oplus \ldots+h_{3}^{\prime}+h_{5}^{\prime}+\ldots .
\end{aligned}
$$

By the inductive hypothesis, $0 \in H_{i}, 2 \leq i \leq n$. Hence,

$$
\begin{aligned}
& A=H_{1} \oplus A_{1}=H_{1}^{\prime}-x_{2} \oplus A_{1}=H_{1}^{\prime} \oplus H_{3}^{\prime} \oplus \ldots+h_{2}^{\prime}+h_{4}^{\prime}+\ldots \\
& B=B_{1}+h_{1}=B_{1}+h_{1}^{\prime}+z_{2}=H_{2}^{\prime} \oplus H_{4}^{\prime} \oplus \ldots+h_{1}^{\prime}+h_{3}^{\prime}+\ldots .
\end{aligned}
$$

This completes the proof.

4. Z-FACTORIZATIONS.

We shall use the term "Z-factorization" when refering to a factorization of the form $G=A \oplus B$, where $A$ and $B$ are Z-sets.

LEMMA 5. Let $G=A \curvearrowright B$ be a Z-factorization of $G$ arising from the series $G=$ $\mathrm{K}_{1} \cdots \mathrm{K}_{2}-\cdots \mathrm{K}_{\mathrm{n}^{-}}<0>$. Then we may choose the coset representatives, $\mathrm{H}_{i}, 1 \leq i \leq \mathrm{n}$, appearing in the expressions for $A$ and $B$ such that $0 \varepsilon H_{i}^{\prime}, 1 \leq i \leq n$, and $h_{i}^{\prime}=0$, $1 \leq \mathrm{i} \leq \mathrm{n}$.

PROOF. We may assume

$$
\begin{aligned}
& A=H_{1} \oplus \mathrm{H}_{3} \mp \ldots+h_{2}+h_{4}+\ldots, \\
& B=H_{2} \Subset H_{4} \subsetneq \ldots+h_{1}+h_{3}+\ldots .
\end{aligned}
$$

By theorem 3 there exist coset representatives $H_{i}^{\prime}, 1 \leq i \leq n$, such that $0 \varepsilon H_{i}^{\prime}$, $1 \leq \mathrm{i} \leq \mathrm{n}$, and

$$
\begin{aligned}
& A=H_{1}^{\prime} \subsetneq H_{3}^{\prime} \mp \ldots+h_{2}^{\prime}+h_{4}^{\prime}+\ldots . \\
& B=H_{2}^{\prime} \mp H_{4}^{\prime} \oplus \ldots+h_{1}^{\prime}+h_{3}^{\prime}+\ldots .
\end{aligned}
$$

Observe that $\mathrm{O} \varepsilon \mathrm{H}_{1}^{\prime}$ क $\mathrm{H}_{3}^{\prime} \mp$. . and $\mathrm{O} \varepsilon \mathrm{H}_{2}^{\prime} \mp \mathrm{H}_{4}^{\prime} \mp \ldots$ Consequently 
$h_{2}^{\prime}+h_{4}^{\prime}+\ldots \varepsilon$ A. Since A is a $Z$-set we have $2\left(h_{2}^{\prime}+h_{4}^{\prime}+\ldots\right) \varepsilon A$. Therefore

$$
2\left(h_{2}^{\prime}+h_{4}^{\prime}+\ldots\right)=\tilde{h}_{2}^{\prime}+\tilde{h}_{3}^{\prime}+\ldots+h_{2}^{\prime} h_{4}^{\prime}+\ldots \text {, }
$$

where $\tilde{h}_{i} \in H_{i}^{\prime}, i=1,3,5, \ldots$. Thus,

$$
h_{2}^{\prime}+h_{4}^{\prime}+\ldots=\tilde{h}_{1}^{\prime}+\tilde{h}_{3}^{\prime}+\ldots .
$$

But $\mathrm{G}=\mathrm{H}_{1}^{\prime} \oplus \mathrm{H}_{2}^{\prime} \oplus \mathrm{H}_{3}^{\prime} \Subset \ldots$ and $0 \in \mathrm{H}_{i}^{\prime}, 1 \leq i \leq \mathrm{n}$. Hence $\mathrm{h}_{2}^{\prime}=\mathrm{h}_{4}^{\prime}=\ldots=0$. Similarly we have $h_{1}^{\prime}=h_{3}^{\prime}=\ldots=0$, so establishing the lemma.

We shall assume throughout the rest of the paper that whenever a Z-factorization $G=A \ni B$ arises from the series $\left.G=K_{1} \supset K_{2}-\ldots \supset K_{n} \supset<0\right\rangle$ the coset representatives have been chosen as in Lemma 5 so that $A=H_{1} \oplus \mathrm{H}_{3} \rightleftarrows \ldots$ and $B=H_{2} \div \mathrm{H}_{4} \notin \ldots$, where $\mathrm{O} \in \mathrm{H}_{i}, 1 \leq i \leq n$.

THEOREM 4. If $G=A(+B$ is a $Z$-factorization of $G$ arising from the series $G=$ $\mathrm{K}_{1}-\mathrm{K}_{2}-\cdots \mathrm{K}_{\mathrm{n}}<0>$ then $\mathrm{K}_{\mathrm{n}}$ is pure in $\mathrm{K}_{\mathrm{n}-1}$.

PROOF. We prove the result for $n$ odd; the proof for $n$ even is similar.

We may assume

$$
\begin{aligned}
& A=H_{1} \oplus \mathrm{H}_{3} \oplus \ldots \mathrm{H}_{n}, \\
& \mathrm{~B}=\mathrm{H}_{2} \oplus \mathrm{H}_{4} \oplus \ldots \ldots+\mathrm{H}_{\mathrm{n}-1},
\end{aligned}
$$

where $0 \in H_{i}, 1 \leq i \leq n$.

Since $K_{n-1}=H_{n-1} \biguplus K_{n}$ we have that $H_{n-1}=B \cap K_{n-1}$ is a Z-set. The result follows from Proposition 1.

LEMMA 6. Let $\mathrm{G}=\mathrm{A} \oplus \mathrm{B}$ be a $\mathrm{Z}$-factorization of $\mathrm{G}$ arising from the series $\mathrm{G}=$ $K_{1}-K_{2} \supset \ldots K_{n}-i<0>$ For $3 \leq i \leq n$ let $\psi_{i}$ be the natural epimorphism with kernel $K_{i}$. Then $\psi_{i}(G)=\psi_{i}(A)+\psi_{i}(B)$ is a Z-factorization of $\psi_{i}(G)$ arising from the series $\psi_{i}(G)=\psi_{i}\left(K_{1}\right) \supset \psi_{i}\left(K_{2}\right)-\ldots D \psi_{i}\left(K_{i-1}\right) \supset \psi_{i}\left(K_{i}\right)$.

PROOF. The result follows from the homomorphic properties of the epimorphisms $\psi_{i}$. THEOREM 5. If $\mathrm{G}=\mathrm{A} \odot \mathrm{B}$ is a Z-factorization of $\mathrm{G}$ arising from the series $\mathrm{G}=$ $K_{1}: K_{2} \cdots K_{n}^{-}<0>$ then $K_{i} / K_{i+1}$ is pure in $K_{i-1} / K_{i+1}, 2 \leq i \leq n-1$.

PROOF. By Lemma 6 a Z-factorization of $\mathrm{G} / \mathrm{K}_{i+1}, 2 \leq i \leq n-1$, arises from the series $G / K_{i+1}=K_{1} / K_{i+1} \frown K_{2} / K_{i+1} \frown \ldots \cdot K_{i-1} / K_{i+1}, K_{i} / K_{i+1-} \frown K_{i+1} / K_{i+1}$. Application of Theorem 4 completes the proof.

5. EXAMPLES.

We now show that the converses of theorems 4 and 5 are false.

EXAMPLE 2. Let $\mathrm{G}$ be a group of type $\left(2^{2}, 2,2\right)$ and let $\mathrm{a}, \mathrm{b}$, and $\mathrm{c}$ of orders $2^{2}, 2$, and 2 respectively generate $G$. Consider the series $G=K_{1}-. \cdot K_{2}>K_{3} \cdot K_{4}^{\prime} \cdot K_{5}$ $=\langle 0\rangle$, where $\left.K_{4}=\langle 2 a\rangle, K_{3}=\langle b\rangle \oplus\langle 2 a\rangle, K_{2}=\langle c\rangle €\right\rangle\langle b\rangle €\langle 2 a\rangle$. Then $K_{i} / K_{i+1} i s$ pure in $\mathrm{K}_{\mathrm{i}-1 / \mathrm{Ki}+1}, 2 \leq 1 \leq 4$. Suppose $\mathrm{u}=\mathrm{i} \oplus \mathrm{B}$ is a $\mathrm{Z}$-factorization arising from the above series. We may assume $A=H_{1} \biguplus H_{3}, B=H_{2} \oplus H_{4}, 0 \varepsilon H_{i}, 1 \leq i \leq 4$. The only possible choices for $\mathrm{H}_{3}$ are $\langle\mathrm{b}\rangle$ and $\langle 2 \mathrm{a}+\mathrm{b}\rangle$, and $\mathrm{H}_{1}$ must have the form $\mathrm{H}_{1}=\{0, \gamma\}, \gamma \neq 0, \gamma \in \mathrm{K}_{2}$. Since $\mathrm{K}_{2}$ contains all the non-zero elements of order 2 , $\gamma$ must be of order $2^{2}$. Thus $r$ has 
the form $r=a+k_{2}$ for some $k_{2} \in K_{2}$. We have that $r \in H_{1} \oplus H_{3}$. Therefore $2 \gamma \in \mathrm{H}_{1} \oplus \mathrm{H}_{3}$. But $2 \gamma \in \mathrm{K}_{2}$. Hence $2 \gamma \in\left(\mathrm{H}_{1} \oplus \mathrm{H}_{3}\right) \cap \mathrm{K}_{2}=\mathrm{H}_{3}$. Depending on the choice for $\mathrm{H}_{3}$, we have that $2 \gamma=b$ or $2 \gamma=2 a+b$. Clearly both cases are impossible and we conclude that for every choice of $H_{3}$ we cannot choose $H_{1}$ such that $A=H_{1}$ † $H_{3}$ is a Z-set.

Example 3 answers the following questions negatively:

If $\mathrm{G}$ is a "bad" group, are all its "good factorizations" (i.e., the factorizations in which at least one factor is periodic) obtained from the variation of Sands' method?

If $\mathrm{G}$ is a "Z-good" group, are all its Z-factorizations obtained from the variation of Sands' method?

EXAMPLE 3. Let $\mathrm{G}$ be a group of type $(p, p, p, p, 2), p$ an odd prime, and let $a_{1}, a_{2}, b_{1}, b_{2}$, and $c$ of orders $p, p, p, p$, and 2 respectively generate $G$. Let $T=$ $\left\langle a_{1}\right\rangle \oplus\left\langle a_{2}\right\rangle \oplus\left\langle b_{1}\right\rangle \oplus\left\langle b_{2}\right\rangle$,

$$
\begin{aligned}
& \left.A^{\prime}=\left(<a_{1}, a_{2}\right\rangle \backslash<a_{2}>\right) \cup<a_{2}+b_{2}>, \\
& \left.B=\left(<b_{1}, b_{2}\right\rangle \backslash\left(\bigcup_{i=1}^{p-1}<b_{1}+i b_{2}>\right)\right) \cup\left(\bigcup_{i=1}^{p-1}<b_{1}+i b_{2}+2 a_{2}>\right) .
\end{aligned}
$$

By Lemma 4 we have that $A^{\prime}$ and $B$ are non-periodic Z-sets and $T=A^{\prime} \oplus B$. Thus $T$ is "Z-bad" and therefore "bad." Consequently, G itself is "bad" [6]. However, in view of Lemma 2, the Sylow 2-subgroup of G, <c>, is "Z-good" so that G is "Z-good" by Lemma 1.

Let $A=A^{\prime} \subsetneq\langle C\rangle$. Clearly $A$ is a periodic Z-set and $\langle c\rangle \subseteq S$, the subgroup of periods of $A$. Let $S \in S$ so that for all $a \in A, a+S \in A$. Then for all $a^{\prime} \varepsilon A^{\prime}$, $a^{\prime}+S \in A$. Thus $a^{\prime}+s=\tilde{a}=\tilde{a}^{\prime}+x, \tilde{a} \varepsilon A, \tilde{a}^{\prime} \in A^{\prime}, x \in\langle c\rangle$. Hence for all $a^{\prime} \varepsilon A^{\prime}$, $a^{\prime}+s-x \in A^{\prime}$ and $s-x$ is a period of $A^{\prime}$. Since $A^{\prime}$ is non-periodic we must have that $s-x=0$, i.e., $s=x \varepsilon<c\rangle$. Therefore $S=\langle c\rangle$.

We have that $G=T \oplus\langle\mathrm{C}\rangle=\mathrm{A} \oplus \mathrm{B}$. Suppose this factorization arises from the series $G=K_{1}-K_{2} \supset \ldots-K_{n^{--}}^{-}<0>$. Since $B$ is non-periodic, $H_{n}=K_{n}$ is not a factor of $B$. Thus there exist transversals $H_{i}$ such that $0 \in H_{i}, 1 \leq i \leq n$, and

$$
\begin{aligned}
& A=H_{n}+H_{n-2}+\ldots, \\
& B=H_{n-1}+H_{n-3}+\cdots .
\end{aligned}
$$

$H_{n}$ is contained in the subgroup of periods of $A$ so that $H_{n}=\langle c\rangle$.

Note that $B \biguplus<c>$ is not a subgroup. Thus $\left.\mathrm{K}_{\mathrm{n}-1} \neq \mathrm{B} \oplus<\mathrm{c}\right\rangle$ and consequently $H_{n-1} \neq B$. But $\left|H_{n-1}\right|$ divides $|B|=p^{2}$. Hence $\left|H_{n-1}\right|=p \cdot H_{n-1}=B \cap K_{n-1}$ implies that $H_{n-1}$ is a $Z$-set. Thus $H_{n-1}$ is a subgroup and we conclude that $B$ is periodic, a contradiction.

Let $G$ be a finite abelian group such that all Sylow subgroups of G are "Z-good." It remains an open question as to whether all "Z-factorizations" of G can be obtained from the variation of Sands' method. 


\section{REFERENCES}

1. HAJOS, G. Uber einfache und merfache Bedeckung des n-dimensionalen Raumes mit einem Wurfelgitter, Math. Zeitschrift 47 (1941), 427-267.

2. HAJOS, G. Sur la factorisation des groupes abelierı, Casopis Pest. Math. Fys. 74 (1950), 157-162.

3. HAJOS, G. Sur le probleme de factorisation des groupes cycliques, Acta Math. Acad. Sci. Hung. 1 (1950), 189-195.

4. REDEI, L. Zwei Luckensatze uber Polynome in endlichen Primkorpern mit Anwendung auf die enlichen Abelschen Gruppen und die Gaussischen Summen, Acta Math 79 (1947), 273-290.

5. REDEI, L. En Beitrag zum Problem der Faktorisation von enlichen Abelschen Gruppen, Acta Math. Acad. Sci. Hung. 1 (1950), 197-207.

6. DE BRUIJN, N.G. On the factorization of finite abelian groups, Indag. Math. Kon. Ned. Akad. Wetensch, Amsterdam 15 (1953), 258-264.

7. DE BRUIJN, N.G. On the factorization of cyclic groups, Indag. Math. Kon. Ned. Akad. hetensch., Amsterdam 15 (1953), 370-377.

8. SANDS, A.D. On the factorization of finite abelian groups I, Acta Math. Acad. Sci. Hung. 8 (1957), 65-86.

9. SANDS, A.D. The factorization of abelian groups I, Quart. J. Math. (2) 10 (1959), $81-91$.

10. SANDS, A.D. On the factorization of finite abelian groups II, Acta Math. Acad. Sci. Hung. 13 (1962), 153-169.

11. SANDS, A.D. The factorization of abelian groups II, Quart. J. Math. (2) 13 (1962), 45-54.

12. OKUDA, C. The Factorization of Abelian Groups, Doctoral Dissertation, The Pennsylvania State University, University Park, PA, 1975. 


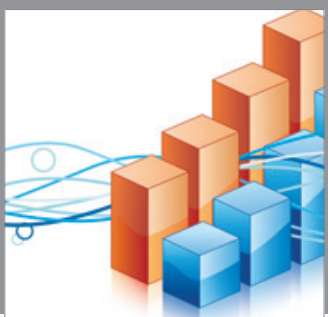

Advances in

Operations Research

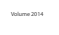

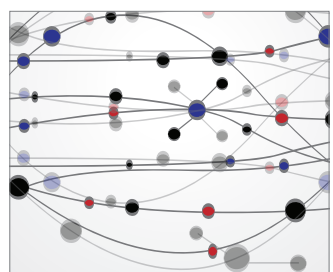

\section{The Scientific} World Journal
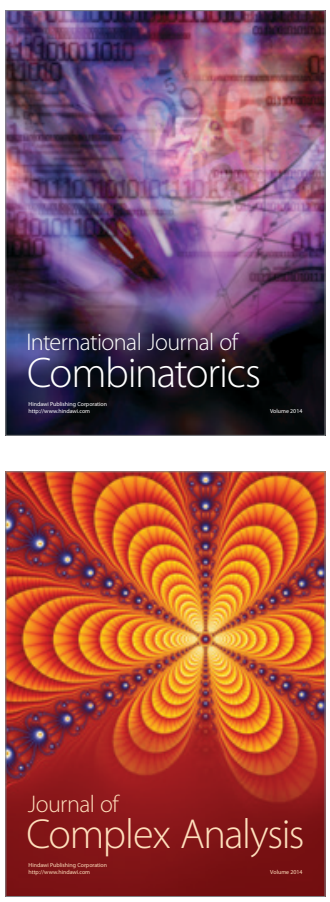

International Journal of

Mathematics and

Mathematical

Sciences
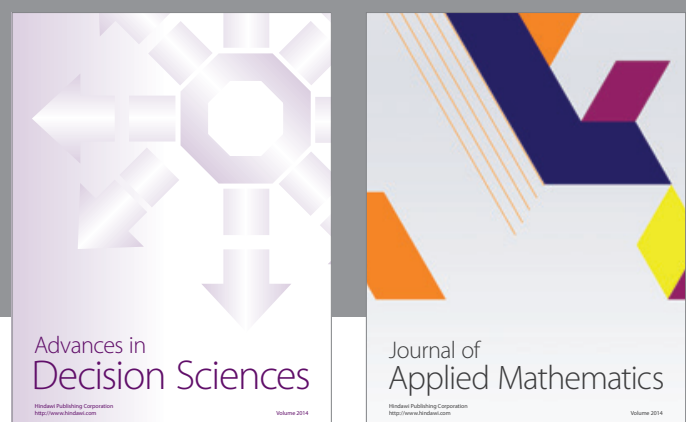

Journal of

Applied Mathematics
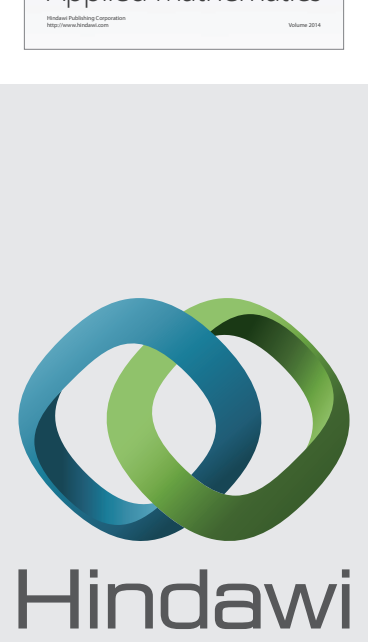

Submit your manuscripts at http://www.hindawi.com
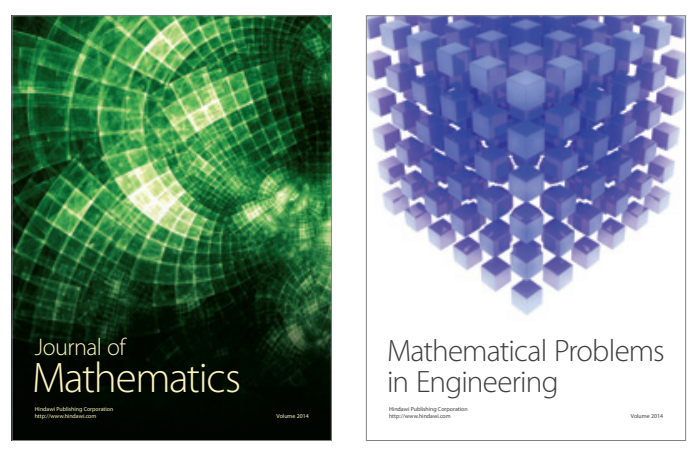

Mathematical Problems in Engineering
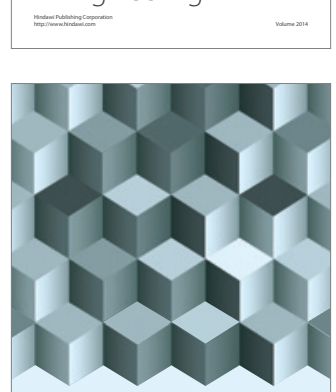

Journal of

Function Spaces
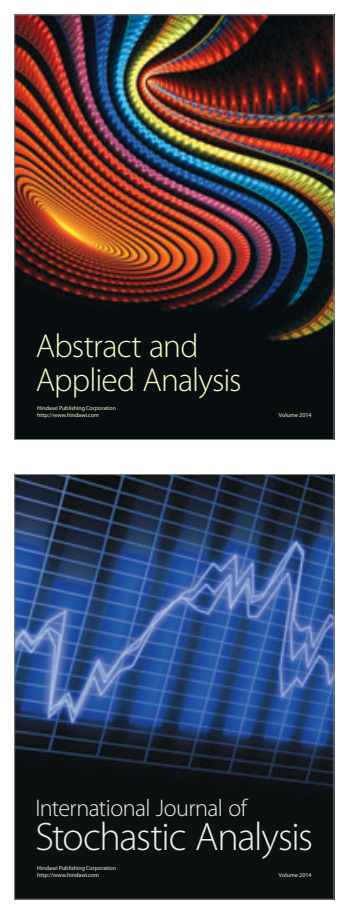

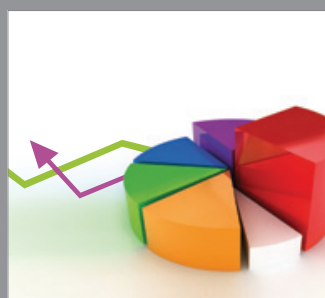

ournal of

Probability and Statistics

Promensencen
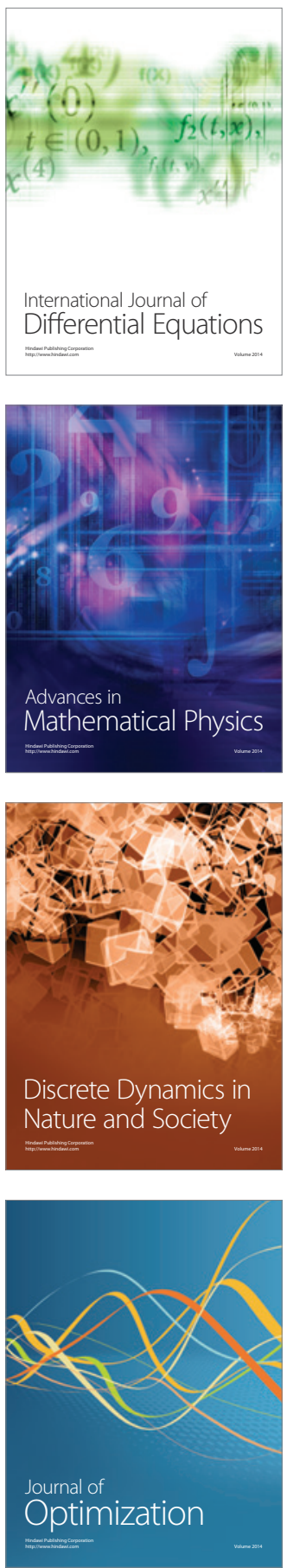